\title{
Effect of iron overload on the severity of liver histologic alterations and on the response to interferon and ribavirin therapy of patients with hepatitis $C$ infection
}

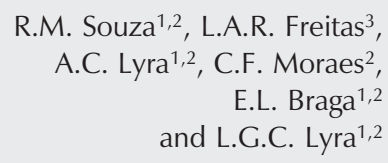

\author{
${ }^{1}$ Serviço de Gastro-Hepatologia, Hospital Prof. Edgard Santos, \\ Universidade Federal da Bahia, Salvador, BA, Brasil \\ ${ }^{2}$ Serviço de Gastro-Hepatologia, Hospital São Rafael, Salvador, BA, Brasil \\ ${ }^{3} \mathrm{CPqGM}$, Fundação Oswaldo Cruz, Salvador, BA, Brasil
}

Correspondence

L.G.C. Lyra

Av. Juracy Magalhães Jr., 1855/501

41940-060 Salvador, BA

Brasil

Fax: +55-71-276-2106 or 399-6266

E-mail: Iglyra@atarde.com.br or

gastro.hepatologia@hsr.com.br

Received September 28, 2004

Accepted September 9, 2005

\begin{abstract}
The objective of the present study was to determine the presence of hepatic iron overload in patients with chronic HCV infection and to correlate it with histologic alterations, HCV genotype and response to therapy. Liver tissue samples from 95 patients with chronic hepatitis $\mathrm{C}$ were divided into two groups: group I, presence of iron overload in hepatic tissue (Perls' staining) and group II, no iron overload. Hepatic iron overload was detected in $30(31.6 \%)$ of 95 patients. Of the 69 patients tested by genotyping, $49(71.01 \%)$ were genotype 1 and 20 (28.99\%) genotype non-1. Iron overload was detected in $14(28.6 \%)$ patients with genotype 1 and in $6(30 \%)$ with genotype non-1 $(\mathrm{P}=$ 0.906). There was a significant difference in fibrosis stage between groups $(\mathrm{P}=0.005)$. In group I $(\mathrm{N}=30)$, one patient had stage $\mathrm{F} 0 / \mathrm{F} 1$ of fibrosis, while in group II $(\mathrm{N}=65), 22(33.8 \%)$ patients had minimal or no fibrosis. Fibrosis stage F2/F3 was observed in $70 \%$ of group I patients compared to $46.2 \%$ of group II. Eighty-five patients were treated with a combination of interferon and ribavirin; 29 of them $(34.1 \%)$ had a sustained virologic response and $8(27.6 \%)$ of them had hepatic iron overload. Iron overload was detected in $18(32.1 \%)$ of the 56 non-responders $(\mathrm{P}=0.73)$. Hepatic iron overload was frequent among patients with chronic hepatitis $\mathrm{C}$ and was associated with a more severe stage of liver fibrosis. There was no association between iron overload and HCV genotype and response to interferon and ribavirin therapy.
\end{abstract}

Elevated iron parameters and mild iron overload are common in the liver of patients with chronic hepatitis $\mathrm{C}$. It has been suggested that ferritin and serum iron might be correlated with liver inflammation and serum markers of fibrogenesis $(1,2)$. Bassett et
Key words

- Hepatitis C infection

- Iron overload

- Genotype

- Inflammatory activity

- Fibrosis

- Antiviral therapy al. (3) showed that iron overload was associated with higher ALT levels and more severe histological findings in $\mathrm{HCV}$-infected chimpanzees. Increased iron stores may stimulate hepatic fibrogenesis, by leading to oxygen free radical injury and/or by inducing the 
production of profibrogenic cytokines such as tumor growth factor $B$ (TGF-B) (2). Of note, there appears to be a discrepancy between the frequency of altered iron parameters in serum and in liver tissue. Riggio et al. (4) found that $40 \%$ of patients had increased iron in serum compared to $10 \%$ in tissue.

The presence of iron overload has been reported to possibly be involved in fibrosis progression and in the development of hepatocellular carcinoma, although the studies are controversial $(5,6)$. Over the last few years there has also been much interest in the role of iron in the outcome of antiviral therapy in patients with chronic HCV infection $(7,8)$.

Several studies have demonstrated that iron overload is associated with lower response rates to interferon- $\alpha$ (IFN- $\alpha$ ) monotherapy. Little is known about whether iron overload also has an impact on the response rate to combination therapy with interferon and ribavirin.

The aims of the present study were to determine the presence of hepatic iron overload in patients with chronic HCV infection and to correlate it with histologic liver alterations, $\mathrm{HCV}$ genotype and the response to combination therapy with interferon and ribavirin.

We evaluated patients who were anti$\mathrm{HCV}$ positive by commercial second- or third-generation ELISA and HCV-RNA positive by PCR (Amplicor, Roche, Indianapolis, IN, USA). Thirty-two patients had been referred to Hospital São Rafael and 63 to Hospital Universitário Prof. Edgard Santos, both in Salvador, BA, Brazil, during the period from 1997 to 2000.

These 95 patients had participated in a previous protocol for the treatment of hepatitis $\mathrm{C}$ and fulfilled the following criteria: 18 -65 years old, ALT level $\geq 1.5$ times the upper normal limit, compensated liver disease, and negative serum markers for HIV and HBV. All subjects had an appropriate amount of liver tissue for analysis and none had been submitted to blood transfusion during the previous 30 days or had other clinical condition that might lead to iron overload.

Eighty-five of 95 patients had completed therapy with interferon using one of the following two schedules: induction therapy with 3 MU IFN- $\alpha$ daily during the first 3 months, followed by treatment three times per week until 1 year was completed, or 3 MU IFN- $\alpha$ three times per week. In both schedules ribavirin was given orally in combination at the dose of $1 \mathrm{~g} /$ day.

Patients were divided into two groups according to the presence (group I) or absence (group II) of iron overload in liver biopsies.

Hepatic tissue fragments were fixed in $10 \%$ formaldehyde, embedded in paraffin and stained with a) hematoxylin and eosin, b) picrosirius red, c) Gomori's silver impregnation, d) PAS with or without diastasis, and e) Perls' staining (Prussian blue reaction). Iron overload was defined as the presence of any amount of iron detected by Perls' staining. Necro-inflammatory activity and stage of liver fibrosis were determined using the METAVIR system (The French METAVIR Cooperative Study Group, 1994).

The Hospital São Rafael Ethics Committee approved the study and the protocol conformed to the ethical guidelines of the 1975 Declaration of Helsinki.

Continuous variables are reported as means \pm SD. Data were analyzed statistically by the chi-square test or Fisher exact test to determine the differences between proportions, and by the Mann-Whitney test, with the level of significance set at $\mathrm{P}<0.05$.

Twenty-four of the 95 patients were females $(25.3 \%)$ and 71 were males $(74.7 \%)$. Mean age was $45.8 \pm 8.7$ years (range 27 $62)$. In most cases the grade of iron overload determined by Perls' staining was mild (1+) or mild to moderate (2+) according to Searle's criteria. According to these data, patients 
were divided into two groups: with or without iron detected in liver tissue.

Group I contained 30 patients $(31.6 \%)$ with iron overload in hepatic tissue and group II consisted of 65 (68.4\%) patients with no iron detected in liver tissue. There was no significant difference between groups in terms of age $(45.4 \pm 8.3$ years in group I $v s$ $45.9 \pm 8.9$ years in group II) or gender.

Histological analysis showed stage F0/ F1 of liver fibrosis in 23 of 95 (24.2\%) patients, stage F2/F3 in 51 (53.7\%) and stage F4 in $21(22.1 \%)$. Necro-inflammatory activity was A0/A1 in 41 of 95 (43.2\%) subjects, A2 in 41 (43.2\%) and A3 in $13(13.6 \%)$. Hepatic steatosis was detected in 53 of 95 $(55.8 \%)$ subjects.

The data in Table 1 show a statistically significant difference in stage of fibrosis between subjects with hepatic iron overload (group I) and patients with no iron detected in the liver (group II) $(\mathrm{P}=0.005)$. In group I, only 1 patient $(3.4 \%)$ had stage F0/F1 of liver fibrosis, while in group II, $33.8 \%$ of patients had minimal or no liver fibrosis (F0/ F1). F2/F3 fibrosis was observed in $70 \%$ of group I patients compared to $46.2 \%$ of group II patients. However, there was no difference between groups in terms of inflammatory activity, ALT levels or presence of hepatic tissue steatosis. A liver section with hepatocellular iron overload in a patient with chronic hepatitis $\mathrm{C}$ without treatment is shown in Figure 1.

$\mathrm{HCV}$ genotype had been determined in 69 individuals; 49 (70\%) were infected with HCV genotype 1, and 20 (30\%) with non-1 HCV genotype. There was no statistically significant difference between groups regarding $\mathrm{HCV}$ genotype.

Among the 85 patients who underwent combination therapy, 29 (34.1\%) were sustained responders while $56(65.9 \%)$ were non-responders or relapsers. Regarding iron overload and sustained virological response, there was also no statistically significant difference between groups.
The present study focuses on a controversial aspect of hepatitis $C$ which is the role of iron in hepatic tissue in patients with

Table 1. Characteristics of patients with hepatitis $C$ infection.

\begin{tabular}{lcccc}
\hline Variables & $\begin{array}{c}\text { Group I } \\
\mathrm{N}(\%)\end{array}$ & $\begin{array}{c}\text { Group II } \\
\mathrm{N}(\%)\end{array}$ & $\mathrm{P}$ & $\begin{array}{c}\text { Total } \\
\mathrm{N}(\%)\end{array}$ \\
\hline Iron overload & $30(31.58)$ & $65(69.42)$ & & 95 \\
HCV genotype & & & 0.9 & \\
$\quad$ Genotype 1 & $14(28.57)$ & $35(71.43)$ & & $49(71.01)$ \\
$\quad$ Genotype non-1 & $6(30)$ & $14(70)$ & & $20(28.99)$ \\
Patients who underwent therapy & & & 0.7 & \\
SVR & $8(27.59)$ & $21(72.41)$ & & $29(34.1)$ \\
Non-SVR & $18(32.14)$ & $38(67.85)$ & & $56(65.9)$ \\
Stage of fibrosis & & & 0.005 & \\
F0-F1 & $1(4.35)$ & $22(95.65)$ & & $23(24.2)$ \\
F2-F3 & $21(41.18)$ & $30(58.82)$ & & $21(22.1)$ \\
F4 & $8(38.1)$ & $13(61.90)$ & & \\
Degree of inflammatory activity & & & 0.47 & \\
A0-A1 & $12(29.27)$ & $29(70.73)$ & & $41(43.1)$ \\
A2 & $12(29.27)$ & $29(70.73)$ & & $41(43.1)$ \\
A3 & $6(46.15)$ & $7(53.85)$ & & $13(13.7)$ \\
\hline SVR = sustained virological response. & & &
\end{tabular}

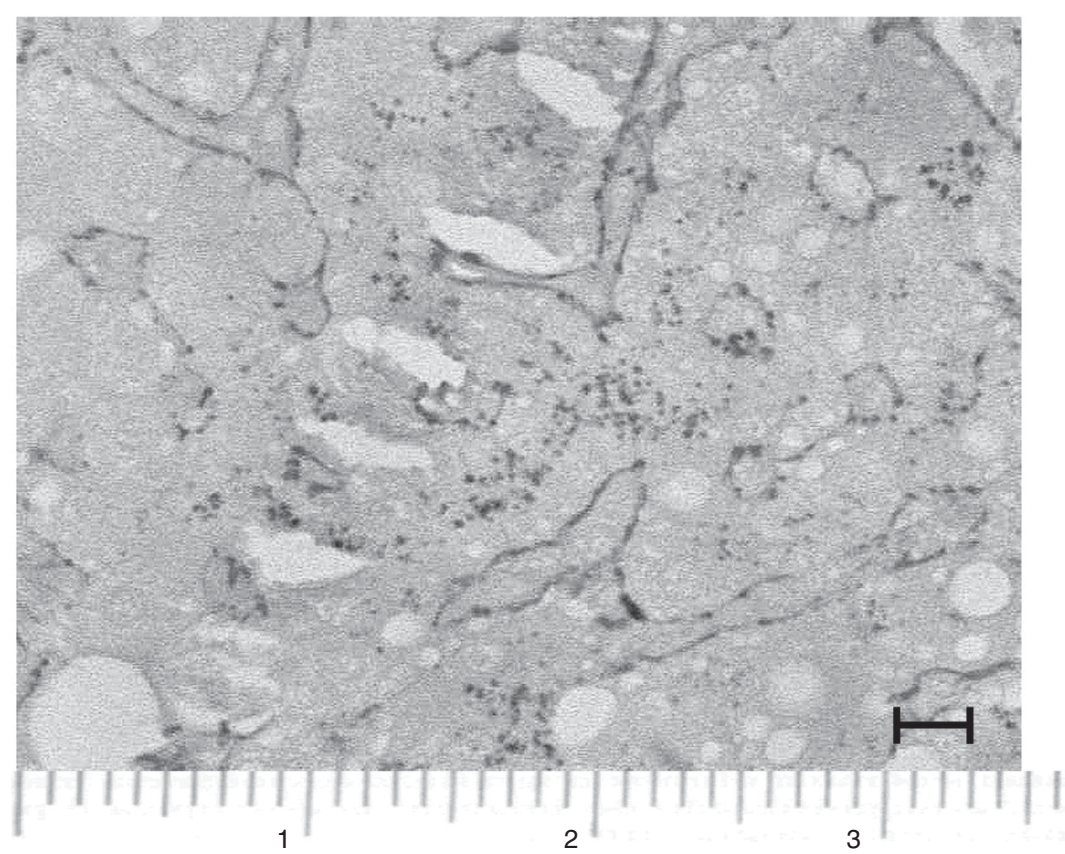

Figure 1. Hepatocellular iron overload in patients with untreated chronic hepatitis C: liver section stained by Perls' method for iron and with sirius red for collagen shows iron granules within the hepatocyte cytoplasm and thin perisinusoidal fibrosis. 400X. Bar $=25 \mu \mathrm{m}$. 
chronic HCV infection. The relationship between HCV infection and serum levels of iron and ferritin was described soon after $\mathrm{HCV}$ was cloned. Later other investigators evaluated the association between iron levels in hepatic tissue and the severity of viral infection or the response to treatment with antiviral drugs $(9,10)$. In our study, in which 95 samples of hepatic biopsy were analyzed, the presence of iron overload in hepatic tissue was observed in $31.6 \%$ of the cases. Other investigators have reported different frequencies of iron overload in patients with chronic hepatitis C. Ikura et al. (11) found that $73 \%$ of 63 patients treated with interferon had iron overload. On the other hand, Riggio et al. (4) found that $10 \%$ of 81 subjects infected with HCV had iron overload. Our results are similar to those reported by Kazemi-Shirazi et al. (12) who evaluated the relationship between the presence of iron and mutation of the hemochromatosis gene in patients with chronic hepatitis C. They detected iron overload in $33.3 \%$ of 149 patients subjected to liver biopsy. In 1999, Hezode et al. (13) reported a frequency of iron overload in $42.1 \%$ of 209 individuals with chronic hepatitis $\mathrm{C}$.

We used Perls' staining to detect iron overload in tissue. A good correlation has been reported between atomic absorption spectroscopy and semi-quantitative determinations in hepatic tissue using Perls' staining $(11,12)$. The biopsy samples from 95 patients were divided into two groups: group I, individuals with iron overload and group II, subjects without iron overload. There was no statistically significant difference by multivariate analysis in terms of clinical characteristics such as age and gender between groups, although there was a predominance of iron overload in male patients. Ikura et al. (11) also observed no statistically significant difference between genders. ALT levels were similar in both groups studied here (mean of $156 \mathrm{vs} 138 \mathrm{U} / \mathrm{L}$ in patients with and without overload, respectively). Kazemi-
Shirazi et al. (12), in 1999, obtained similar results when they studied iron status and the hemochromatosis mutation in 114 patients with chronic hepatitis C. Di Bisceglie et al. (9) found a difference between serum AST and ferritin levels but did not identify any difference between ALT levels and hepatic iron concentration.

$\mathrm{HCV}$ genotype was determined in 69 of our 95 patients. Most of them (70\%) were infected with $\mathrm{HCV}$ genotype 1 and $30 \%$ with genotypes 2 and 3 . The higher frequency of HCV genotype 1 has been reported for most Brazilian regions (14). There was no statistically significant difference by multivariate analysis between the patients with iron overload in hepatic tissue and HCV genotype, in spite of the fact that some studies have reported a higher frequency of iron overload in patients infected with HCV genotype 1 (15).

It is not clear if the presence of iron overload interferes with the response of the liver to the therapeutic schedules with interferon and ribavirin. Some researchers considered iron overload to be a factor contributing to a decreased response to treatment $(16,17)$. Later investigations reported contradictory results. Currently, there is no consensus as to the role of iron overload in the response to treatment of chronic hepatitis $\mathrm{C}$ or if there may be a subgroup of patients in which iron overload interferes with therapy. Recently, some investigators concluded that hepatic iron concentration does not predict the response to standard and pegylated interferon/ribavirin therapy in patients with chronic hepatitis C (18). Our data did not detect a difference between patients with iron overload that achieved a virological sustained response $(28.57 \%)$ and non-responders $(32.14 \%)$.

Hepatic fibrosis develops as a result of the response of the liver to chronic injury. Iron stores may stimulate hepatic fibrogenesis, either by leading to oxygen free radical injury and/or by inducing the production of profibrogenic cytokines such as TGF- $B$. This 
could contribute to the development of hepatic cirrhosis and hepatocellular carcinoma. Our results are consistent with reports which found that iron overload might be associated with increased liver fibrosis $(19,20)$.

Hepatic iron overload was observed in $31.6 \%$ of patients with hepatitis $\mathrm{C}$, espe- cially males. A greater iron accumulation was associated with a more severe stage of liver fibrosis, but there was no relationship between iron overload and response to treatment with interferon and ribavirin. However, sample size might have been a limiting factor for definitive conclusions.

\section{References}

1. Pietrangelo A (1998). Iron, oxidative stress and liver fibrogenesis. Journal of Hepatology, 28 (Suppl 1): 8-13.

2. Casaril M, Stanzial AM, Tognella P et al. (2000). Role of iron load on fibrogenesis in chronic hepatitis C. Hepato-Gastroenterology, 47: 220-225.

3. Bassett SE, Di Bisceglie AM, Bacon BR et al. (1999). Effects of iron loading on pathogenicity in hepatitis $C$ virus-infected chimpanzees. Hepatology, 29: 1884-1892.

4. Riggio O, Montagnese F, Fiore P et al. (1997). Iron overload in patients with chronic viral hepatitis: how common is it? American Journal of Gastroenterology, 92: 1298-1301.

5. Chapoutot C, Esslimani M, Joomaye Z et al. (2000). Liver iron excess in patients with hepatocellular carcinoma developed on viral C cirrhosis. Gut, 46: 711-714.

6. Ganne-Carrie N, Christidis C, Chastang C et al. (2000). Liver iron is predictive of death in alcoholic cirrhosis: a multivariate study of 229 consecutive patients with alcoholic and/or hepatitis $C$ virus cirrhosis: a prospective follow up study. Gut, 46: 277-282.

7. Olynyk J, Reddy K, Di Bisceglie A et al. (1995). Hepatic iron concentration as a predictor of response to interferon alpha therapy in chronic HCV. Gastroenterology, 108: 1104-1109.

8. Hayashi H, Takikawa T, Nishimura N et al. (1994). Improvement of serum aminotransferase levels after phlebotomy in patients with chronic active hepatitis $\mathrm{C}$ and excess hepatic iron. American Journal of Gastroenterology, 89: 986-988.

9. Di Bisceglie AM, Axiotis C, Hoofnagle $\mathrm{J}$ et al. (1992). Measurements of iron status in patients with chronic hepatitis. Gastroenterology, 102: 2108-2113.

10. Arber N, Konikoff F \& Moshkowitz M (1994). Increased serum iron and iron saturation without liver iron accumulation distinguish chronic hepatitis C from other chronic liver diseases. Digestive Diseases and Sciences, 39: 2656-2659.

11. Ikura $\mathrm{Y}$, Morimoto $\mathrm{H}$, Johmura $\mathrm{H}$ et al. (1996). Relationship between hepatic iron deposits and response to interferon in chronic hepatitis
C. American Journal of Gastroenterology, 91: 1367-1373.

12. Kazemi-Shirazi L, Datz C, Maier-Dobersberger T et al. (1999). The reaction of iron status and hemochromatosis gene mutations in patients with chronic hepatitis C. Gastroenterology, 116: 127-134.

13. Hezode C, Cazeneuve C, Coué O et al. (1999). Liver accumulation in patients with chronic active hepatitis C: prevalence and role of hemochromatosis gene mutations and relationship with hepatic histology lesions. Journal of Hepatology, 31: 979-984.

14. Busek S \& Oliveira G (2003). Molecular epidemiology of the hepatitis C virus in Brazil. Genetics and Molecular Research, 2: 117-123.

15. Gehrke SG, Stremmel W, Mathes I et al. (2003). Hemochromatosis and transferrin receptor gene polymorphisms in chronic hepatitis $\mathrm{C}$ : impact on iron status, liver injury and HCV genotype. Journal of Molecular Medicine, 81: 780-787.

16. Kageyama F, Kobayashi $Y$, Murohisa G et al. (1998). Failure to respond to interferon-alpha $2 a$ is associated with increased hepatic iron levels in patients with chronic hepatitis C. Biological Trace Element Research, 64: 185-196.

17. Distante S, Bjoro K, Hellum KB et al. (2002). Raised serum ferritin predicts non-response to interferon and ribavirin treatment in patients with chronic hepatitis C infection. Liver, 22: 269-275.

18. Hofer H, Osterreicher C, Jessner W et al. (2004). Hepatic iron concentration does not predict response to standard and pegylated INF/ribavirin therapy in patients with chronic hepatitis C. Journal of Hepatology, 40: 1018-1022.

19. Angelucci E, Muretto P, Nicolucci A et al. (2002). Effects of iron overload and hepatitis $C$ virus positivity in determining progression of liver fibrosis in thalassemia following bone marrow transplantation. Blood, 100: 17-21.

20. Ardalan FA, Osquei MR, Toosi MN et al. (2004). Synergic effect of chronic hepatitis $C$ infection and beta thalassemia major with marked hepatic iron overload on liver fibrosis: a retrospective cross-sectional study. BMC Gastroenterology, 4: 17. 